\title{
Erratum: "Electron Pre-acceleration at Nonrelativistic High-Mach-number Perpendicular Shocks" (2017, ApJ, 847, 71)
}

\author{
Artem Bohdan $^{1}$ (D), Jacek Niemiec ${ }^{1}$ (D), Oleh Kobzar $^{1}$ (D), and Martin Pohl ${ }^{2,3}$ (D) \\ ${ }^{1}$ Instytut Fizyki Jạdrowej PAN, ul. Radzikowskiego 152, 31-342 Kraków, Poland \\ ${ }^{2}$ Institute of Physics and Astronomy, University of Potsdam, D-14476 Potsdam, Germany \\ ${ }^{3}$ DESY, D-15738 Zeuthen, Germany \\ Received 2019 July 3; published 2019 July 25
}

In the published article, an error was introduced in Table 2. The value presented in the last column should be $k T / m c^{2}$ but was by mistake not normalized by $m c^{2}$. The numerical values should be larger by a factor of four. The revised Table 2 provides the correct numbers.

The last sentence in the third paragraph of Section 3.4.5 should be corrected accordingly, namely, the electron temperatures are higher by a factor 18-33 than those predicted by the Rankine-Hugoniot jump conditions if only electrons are considered, indicating that significant bulk heating has occurred (compare Matsumoto et al. 2012).

This result does not affect the other conclusions of our paper.

Table 2

Downstream Spectra Parameters

\begin{tabular}{llccc}
\hline \hline Run & $\varphi$ & $\beta_{\mathrm{p}}$ & $\begin{array}{c}\text { NTEF } \\
(\%)\end{array}$ & $\begin{array}{c}N_{e}(\gamma>3) \\
(\%)\end{array}$ \\
\hline A1 & & 0.0005 & $0.2 \pm 0.1$ & 0.1 \\
A2 & $0^{\circ}$ & 0.5 & $0.7 \pm 0.1$ & 0.06 \\
\hline B1 & $0^{\circ}$ & 0.0005 & $0.2 \pm 0.1$ & 0.11 \\
B2 & $45^{\circ}$ & 0.5 & $0.5 \pm 0.1$ & 0.17 \\
\hline C1 & $45^{\circ}$ & 0.0005 & $4 \pm 1$ & 0.22 \\
C2 & $90^{\circ}$ & 0.5 & $7 \pm 1$ & 0.12 \\
\hline
\end{tabular}

Note. Comparison of the characteristics of the energy distribution of electrons in the downstream region of all six simulated shocks. NTEF denotes the nonthermal electron fraction.

\section{ORCID iDs}

Artem Bohdan (10) https://orcid.org/0000-0002-5680-0766

Jacek Niemiec (ib https://orcid.org/0000-0001-6036-8569

Oleh Kobzar (iD https://orcid.org/0000-0001-6956-5884

Martin Pohl (1) https://orcid.org/0000-0001-7861-1707

\section{Reference}

Matsumoto, Y., Amano, T., \& Hoshino, M. 2012, ApJ, 755, 109 